\title{
Long non-coding RNA linc00239 promotes malignant behaviors and chemoresistance against doxorubicin partially via activation of the PI3K/Akt/mTOR pathway in acute myeloid leukaemia cells
}

\author{
YUTING YANG $^{1 *}$, WENSHU DAI $^{2 *}$, YINGWEI SUN $^{3}$ and ZIYI ZHAO ${ }^{4}$ \\ ${ }^{1}$ Department of Scientific Research, The Teaching Hospital of Chengdu University of Traditional Chinese Medicine, \\ Chengdu, Sichuan 610072, P.R. China; ${ }^{2}$ School of Biomedical Science, The University of Queensland, \\ Brisbane St Lucia, QLD 4072, Australia; ${ }^{3}$ Department of Pharmacy and ${ }^{4}$ Central Laboratory, \\ The Teaching Hospital of Chengdu University of Traditional Chinese Medicine, \\ Chengdu, Sichuan 610072, P.R. China
}

Received July 8, 2018; Accepted January 18, 2019

DOI: 10.3892/or.2019.6991

\begin{abstract}
Long non-coding RNAs (lncRNAs) are known to be involved in the processes of tumourigenesis and malignant behaviours in many types of cancer, including acute myeloid leukaemia (AML). Accumulating evidence has revealed that novel lncRNAs exerted critical roles in these processes. In the present study, we investigated the effects of lncRNA linc00239 (NR_026774.1), which is 662 nucleotides (nt) in length and was found to be upregulated in AML patients, on malignant behaviours and chemosensitivity in AML cells, including KG-1 and HL-60. linc00239 expression was detected in KG-1 and HL-60 cells by quantitative PCR and northern blotting, and it was found that linc00239 is detectable by both of these assays. After knockdown or overexpression of linc00239 in AML cells, the results revealed that the presence of linc00239 promoted proliferation, colony formation and migration ability. Furthermore, the presence of linc00239 increased chemoresistance to doxorubicin in AML cells partially by preventing doxorubicin-induced apoptotic cell death. It was also determined that the presence of linc00239 was related to activation of the phosphatidylinositol 3-kinase (PI3K)/Akt/mammalian target of rapamycin (mTOR) pathway. Inhibition of PI3K/Akt/mTOR using $1 \mu \mathrm{M}$ NVP-BEZ235
\end{abstract}

Correspondence to: Dr Ziyi Zhao, Central Laboratory, The Teaching Hospital of Chengdu University of Traditional Chinese Medicine, 39 Shierqiao Road, Jinniu, Chengdu, Sichuan 610072, P.R. China

E-mail: zhaoziyi925@163.com

${ }^{*}$ Contributed equally

Key words: long non-coding RNA, acute myeloid leukemia, chemoresistance, PI3K/Akt/mTOR, linc00239
(BEZ) abolished the inhibitory effect of linc00239 on chemosensitivity and the preventative effect on doxorubicin-induced cell death. Collectively, our data revealed that linc00239 is a novel tumour promoter in AML cells and indicated that it is a potential therapeutic target.

\section{Introduction}

Acute myeloid leukaemia (AML) is a heterogeneous haematopoietic malignancy, which is well-known for the rapid accumulation and malignant proliferation of immature myeloid progenitors in the bone marrow (BM) and peripheral blood (PB) (1). For decades, without efficient therapeutic strategy, AML quickly led to poor prognosis and became fatal. Over the last few decades, AML treatment has improved in risk assessment, post-remission chemotherapy and haematopoietic stem-cell transplantation (2,3). The phosphoinositide 3-kinase, AKT, and mammalian target of rapamycin (PI3K/AKT/mTOR) signalling network controls proliferation, differentiation, and survival of haematopoietic cells $(4,5)$. The fact that in $50-80 \%$ of AML cases, the PI3K/AKT/mTOR pathway is abnormally activated indicates that the $\mathrm{PI} 3 \mathrm{~K} / \mathrm{AKT} / \mathrm{mTOR}$ pathway is a promising therapeutic target $(6,7)$, and many agents have been developed. LY294002, which directly targets PI3K, has revealed strong cytotoxic effects in preclinical AML models (8-10). Ribavirin, an eIF4E inhibitor, was studied in preclinical models and in a pilot study and presents efficiency in $30 \%$ of AML patients $(11,12)$. Aberrant activation of $\mathrm{AKT}$ is the most common frequent cause in AML, and dual $\mathrm{PI} 3 \mathrm{~K} / \mathrm{mTOR}$ inhibitors (P-103 and BEZ235), mTOR 1/2 inhibitors (OSI-027 and PP242), and AKT inhibitors (perifosine) were also investigated in preclinical models (13-17).

Long non-coding RNAs (lncRNAs) are a class of RNA molecules $>200$ nucleotides. They are transcribed and not protein coded. A large number of this class of RNA molecules has been identified, numbering $>58,000(18,19)$. Accumulating evidence has revealed that IncRNAs play critical roles not 
only in physiological processes in normal cells but also in a multitude of biological processes that are central in tumorigenesis and the progression of cancer $(18,20)$, including in AML. Wu et al reported that HOX transcript antisense intergenic RNA (HOTAIR) was overexpressed in AML and was a promising biomarker of poor prognosis (21). By performing large-scale sequencing, it was determined via evaluation of the IncRNA profile that it is possible to provide valuable information for improved risk stratification of AML patients (22).

IncRNAs are reported to regulate the PI3K/AKT/mTOR pathway in several types of cancer cells. Highly upregulated in liver cancer (HULC) was revealed to act as an oncogene in gliomas (23). The upregulated HULC promoted malignant cell behaviours, including proliferation, migration and invasion via activation of the PI3K/AKT/mTOR pathway in glioma cells (23). A newly identified lncRNA ENST00113, which is most upregulated in atherosclerosis, regulated proliferation, survival and migration by activating the PI3K/AKT/mTOR pathway, potentially by inhibiting the process of phosphorylation (24). IncRNA LUNAR1 was reported to play a central role in the interplay of NOTCH1-IGF-1 interactions via activation of the PI3K/AKT/mTOR pathway in AML cells (25). Accordingly, these data revealed that lncRNAs are tightly associated with activation or inactivation of the PI3K/AKT/mTOR pathway and thus play a regulatory role in the malignant behaviours of several types of cancers, including AML.

Long intergenic non-protein coding RNA 239 (LINC00239) is a newly identified IncRNA that has been revealed to be aberrantly expressed in AML (26), without knowledge of its exact role in AML. In the present study, the fundamental role of LINC00239 in human AML cells was examined and the potential role in regulating malignant behaviours via the $\mathrm{PI} 3 \mathrm{~K} / \mathrm{AKT} / \mathrm{mTOR}$ pathway was investigated.

\section{Materials and methods}

Cell culture and treatment. The AML cell lines HL-60 (FAB M2) and KG-1 (erythroleukemia-FAB M6) were obtained from the American Type Culture Collection (ATCC; Manassas, VA, USA). Cells were maintained in RPMI-1640 medium supplemented with $10 \%$ heat-inactivated fetal bovine serum (FBS) (both from Gibco; Thermo Fisher Scientific, Inc., Waltham, MA, USA), $1 \%$ antibiotic-antimycotic mixture (Thermo Fisher Scientific, Inc.) in a humidified, $37^{\circ} \mathrm{C}$ and $5 \% \mathrm{CO}_{2}$ atmosphere. Medium was half-refreshed every 3 days.

Doxorubicin and dual pan-PI3K/mTOR kinase inhibitor dactolisib (NVP-BEZ235) were purchased from (Sigma-Aldrich; Merck KGaA, Darmstadt, Germany). For the evaluation of chemosensitivity to doxorubicin, a range concentration from 0.5 to $100 \mu \mathrm{M}$ was incubated with KG-1 or HL-60 cells for $24 \mathrm{~h}$, respectively. For inhibition of the PI3K/AKT/mTOR pathway, KG-1 or HL-60 cells were pretreated with $0.5 \mathrm{Mm}$ NVP-BEZ235 for $6 \mathrm{~h}$.

$R T-q P C R$. Total RNA was isolated from culture cell using TRIzol $^{\circledR}$ (Invitrogen; Thermo Fisher Scientific, Inc.) according to the manufacturer's instructions. Reverse transcription of RNA was performed following the instructions of the MBI Fermentas kit (Fermentas; Thermo Fisher Scientific, Inc.). Reaction conditions were as follows: $70^{\circ} \mathrm{C}$ for $10 \mathrm{~min}$, ice bath for $2 \mathrm{~min}, 42^{\circ} \mathrm{C}$ for $60 \mathrm{~min}$ and $70^{\circ} \mathrm{C}$ for $10 \mathrm{~min}$. The cDNA was stored in a $-80^{\circ} \mathrm{C}$ freezer. The reaction system for qPCR was prepared following the instructions of the kit (Fermentas; Thermo Fisher Scientific, Inc.). The primer sequences were as follows: linc00239, forward 5'-AGGTACCAGCTGGGATGT TG-3' and reverse 5'-CATGACCGTCTTCCTGTGTG-3'; U6, forward 5'-GTGCTCGCTTCGGCAGCACA-3' and reverse 5'-AAAATATGGAACGCTTCA-3'; $\beta$-actin, forward 5'-CAT GTACGTTGCTATCCAGGC-3' and reverse 5'-CTCCTTAAT GTCACGCACGAT-3'; GAPDH, forward 5'-GGAGCGAGA TCCCTCCAAAAT-3' and reverse 5'-GGCTGTTGTCAT ACTTCTCATGG-3'. The reaction conditions were as follows: pre-denaturation at $98^{\circ} \mathrm{C}$ for $2 \mathrm{~min}$, denaturation at $98^{\circ} \mathrm{C}$ for $5 \mathrm{sec}$, annealing and extension at $60^{\circ} \mathrm{C}$ for $1 \mathrm{~min}, 35$ cycles in total. snoU6, $\beta$-actin and GAPDH were used as the internal references. The expression of the genes were calculated using relative quantitative measurement, where $2^{-\Delta \Delta C q}$ represented the relative expression of each target gene (27). Each experiment was repeated for 3 times.

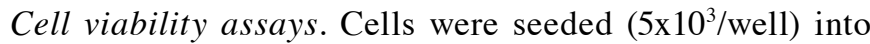
96-well plates overnight. After treatment with doxorubicin (0.5-100 $\mu \mathrm{M})$ for $24 \mathrm{~h}, 10 \mu \mathrm{l}$ tetrazolium salt Cell Counting Kit-8 (CCK-8) (Nanjing KeyGen Biotech Co., Ltd., Nanjing, China) was added to each well (final volume ratio, $10 \%$ ). Optical density (OD) was assessed at a wavelength of $450 \mathrm{~nm}$ (OD450).

Cell cycle distribution. Cells were pelleted and washed with pre-cold phosphate-buffered saline (PBS) 3 times. Cells were fixed with $1 \mathrm{ml}$ of $75 \%$ ethyl alcohol, stored overnight at $4^{\circ} \mathrm{C}$, washed with PBS 2 times and $100 \mu 1$ RNase A and $400 \mu 1$ propidium iodide (PI; Sigma-Aldrich; Merck KGaA) were added in the dark and incubated for $30 \mathrm{~min}$ at room temperature. After 30 -min incubation at $4^{\circ} \mathrm{C}$, flow cytometric analysis was performed using the FACS LSR II flow cytometer (BD Biosciences, Franklin Lakes, NJ, USA).

Colony formation on soft agar. KG-1 or HL- 60 cells $\left(5 \times 10^{3}\right)$ were suspended in serum-free RPMI-1640 medium and dissolved in $0.3 \%$ low-melting agarose (Sigma-Aldrich; Merck KGaA), supplemented with $10 \%$ FBS and a $1 \%$ antibiotic-antimycotic mixture. Cells were allowed to grow for 14 days and colonies were identified as having $>50$ cells. Cells were visualized using an Olympus CX41 microscope (Olympus Corp., Tokyo, Japan).

Migration. Transwell permeable plates with $8.0 \mu \mathrm{M}$ pores (Corning Incorporated Life Sciences, Corning, NY, USA) were employed to analyze cell migration. In the bottom chamber, $500 \mu \mathrm{l}$ of serum-free RPMI-1640 medium was added supplemented with 5\% FBS. In the upper chamber, $5 \times 10^{4}$ cells were added suspended in serum-free medium. After $24 \mathrm{~h}$ of incubation, the amount of viable migrated cells was determined by counting using trypan blue staining. Cells were visualized using an Olympus CX41 microscope (Olympus Corp.).

CFSE/PI staining. Cells were washed in serum-free RPMI-1640 medium 3 times and suspended in $500 \mu \mathrm{l}$ of fresh medium. Carboxyfluorescein diacetate succinimidyl ester (CFSE) fluorescent dye $(100 \mu \mathrm{l})(5 \mu \mathrm{mol} / \mathrm{l})$ was added and 
incubated at $37^{\circ} \mathrm{C}$ for $30 \mathrm{~min}$. Then, the cells were washed with fresh medium 3 times. Subsequently, $24 \mathrm{~h}$ later, cells were collected and suspended in $500 \mu \mathrm{l}$ fresh medium and incubated with $5 \mu \mathrm{g} / \mathrm{ml}$ PI at room temperature for $10 \mathrm{~min}$. Flow cytometry was performed using the FACS LSR II flow cytometer (BD Biosciences). Analysis of results was performed using FlowJo 7.6 software (Tree Star, Inc., Ashland, OR, USA). The experiments were repeated at least 3 times.

Annexin V-FITC/PI staining. KG-1 or HL-60 cells were washed with $1 \mathrm{ml}$ of ice-cold PBS 3 times. Cells were suspended with $100 \mu \mathrm{l}$ of binding buffer, $5 \mu \mathrm{l}$ of Annexin V-FITC (BDBiosciences) and $10 \mu \mathrm{l}$ of PI (Sigma-Aldrich; Merck KGaA) for 10 min avoiding light at room temperature. Then, FITC and PI signals were assessed using FACS LSR II flow cytometer (BD Biosciences). Analysis of results was performed using FlowJo 7.6 software (Tree Star, Inc.). The experiments were repeated at least 3 times.

TUNEL staining. Cells were fixed with $4 \%$ paraformaldehyde at room temperature for $10 \mathrm{~min}$. After 3 washes with PBS, cells were permeabilized with $0.1 \%$ Triton $\mathrm{X}-100$ in PBS for $10 \mathrm{~min}$ at room temperature. Cells were stained with In Situ Cell Death Detection Kit (Roche Diagnostics, Basel, Switzerland) following the manufacturer's instructions. Cells were visualized under an X71 (U-RFL-T) fluorescence microscope (Olympus, Melville, NY, USA) and analyzed with ImageJ software (version 1.46; National Institutes of Health, Bethesda, MD, USA).

Western blotting. For the extraction of total protein from KG-1 or HL-60 cells, $100 \mu \mathrm{l}$ of lysis buffer containing 1\% NP-40, $100 \mathrm{mM}$ Tris- $\mathrm{HCl} \mathrm{pH} 7.5,150 \mathrm{mM} \mathrm{NaCl}$ and $5 \mathrm{mM}$ EDTA was employed. The protein concentration was measured using Pierce BCA Protein Assay Kit (Thermo Fisher Scientific, Inc.). Total protein $(20 \mu \mathrm{g})$ was fractionated in a $10 \%$ SDS gel and transferred to nitrocellulose membranes. The membranes were blocked in PBS with $0.25 \%$ Tween-20 (PBS-T) containing $5 \%$ skim milk for $1 \mathrm{~h}$ at room temperature. The blocked membranes were incubated with primary antibodies for $2 \mathrm{~h}$ at room temperature. The primary antibodies used were as follows: anti- $\gamma$-H2A.X antibody diluted in 1:1,000 (cat. no. ab2893), anti-histone H2A.X antibody diluted in $1: 2,500$ (cat. no. ab11175), anti- $\beta$-actin antibody diluted in 1:5,000 (cat. no. ab8226), anti-AKT (phospho S473) antibody diluted in 1:1,000 (cat. no. ab18206), anti-pan-AKT antibody diluted in 1:1,000 (cat. no. ab8805), anti-mTOR (phospho S2448) diluted in 1:500 (cat. no. ab109268), anti-mTOR diluted in 1:1,000 (cat. no. ab2732) and anti-GAPDH antibody diluted in 1:5,000 (cat. no. ab8245). All antibodies were purchased from Abcam (Cambridge, UK). After 3 washes with PBS-T, the membranes were incubated with the corresponding following secondary antibodies: HRP-labeled goat anti-mouse IgG antibody diluted in 1:5,000 (cat. no. ab97040) or goat anti-rabbit IgG antibody diluted in 1:5,000 (cat. no. ab7090) for $2 \mathrm{~h}$ at room temperature. Protein levels were detected after incubation with SuperSignal Wester Femto Maximum Sensitivity Substrate (Thermo Fisher Scientific, Inc.). Blots were imaged and analyzed with Quantity One (Bio-Rad Laboratories, Inc., Hercules, CA, USA).
Statistical analysis. All data were presented as the means \pm SD (SD). Student's t-test was performed to assess statistical significance. The Wilcoxon signed-rank test was used to compare paired samples, whereas Kruskal-Wallis and Mann-Whitney $\mathrm{U}$ tests were used to compare different groups followed by the Tukey's post hoc test. P-values $<0.05$ were considered to indicate a statistically significant difference.

\section{Results}

Confirmation of the existence of IncRNA linc00239 in the $A M L$ cell lines $K G-1$ and $H L-60$. In order to identify the expression levels of linc00239 in the AML cell lines KG-1 and HL-60, RT-qPCR was performed, and the data were normalized to $\beta$-actin, GAPDH or snoU6 RNA. As revealed in Fig. 1A, the expression of linc00239 was detected in both KG-1 and HL-60 cells, and KG-1 presented an evidently higher expression compared to HL-60. Northern blotting also confirmed the existence of linc00239 in both of these cell lines (Fig. 1B). shRNA targeted to linc00239 (shlinc00239) or scrambled shRNA (shScrambled) was transiently transfected into KG-1 cells for linc00239 knockdown. linc00239 expression vector (linc00239) or empty vector (vector) was transiently transfected into HL-60 cells for linc00239 overexpression analysis. Efficient overexpression by expressing vector or knockdown by shlinc00239 transient transfection was confirmed by RT-qPCR (Fig. 1C).

Effects of linc00239 on cell viability, cell cycle distribution, colony formation and migration. The accumulating evidence indicating that IncRNAs may tightly regulate tumour development encouraged us to investigate the exact role of linc00239 in AML cells $(28,29)$. By performing a CCK-8 assay and PI staining, followed by a flow cytometric assay, the results revealed that although linc00239 knockdown slightly affected cell viability and cell cycle distribution in KG-1 cells, linc00239 overexpression significantly promoted cell viability potentially by promoting the cell cycle by decreasing the $G_{1} / G_{0}$ phase in HL-60 cells (Fig. 2A and B). The effects of linc 00239 on colony formation and migration were further detected to illustrate the role of linc00239 in the regulation of malignant behaviours in AML cells. As revealed in Fig. 2C and D, in both KG-1 and HL-60 cells, the presence of linc00239 positively regulated colony formation and migration.

linc00239 regulates chemoresistance to doxorubicin and exerts a protective effect against apoptotic cell death. Various lncRNAs have been reported to regulate chemosensitivity in cancer cells to therapeutic agents, including doxorubicin, by modulating DNA damage response pathways $(30,31)$. This encouraged us to investigate the role of linc00239 in the chemosensitivity to doxorubicin, which is a commonly used therapeutic agent. By exposure to a concentration range of doxorubicin, relative cell viability was assessed by performing a CCK-8 assay. In KG-1 cells, knockdown of linc00239 significantly decreased the $\mathrm{IC}_{30}$ and $\mathrm{IC}_{50}$ concentrations of doxorubicin (Fig. 3A and B, upper panel). After $1.75 \mu \mathrm{M}$ treatment of doxorubicin, the rate of cell death detected by CFSE/PI double staining illustrated consistently that knockdown of linc00239 significantly increased the cell death rate. In HL-60 


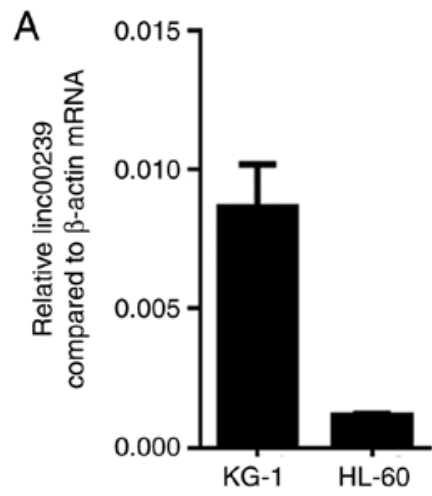

B

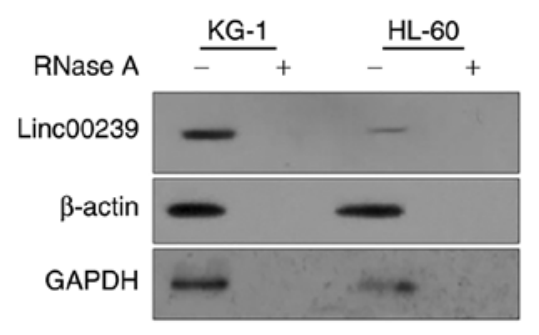

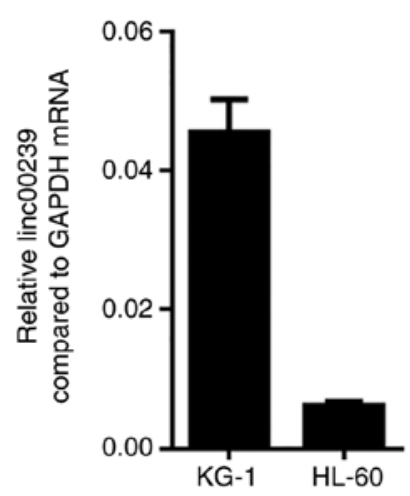

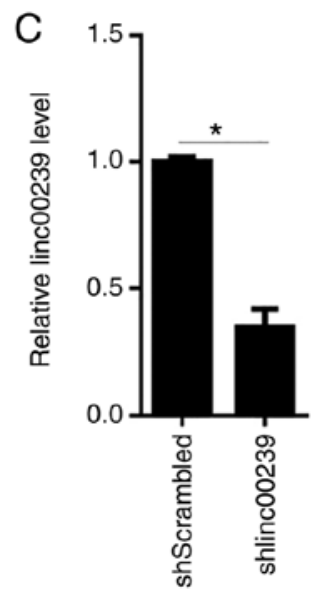

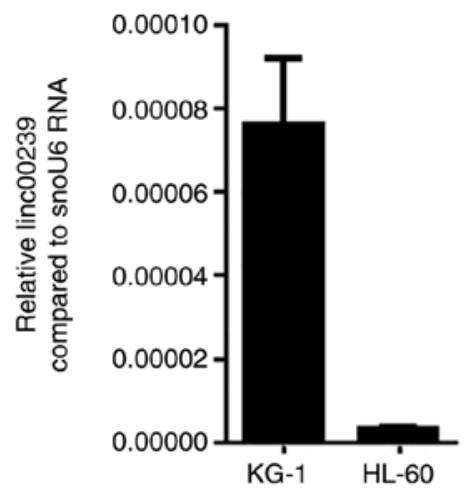

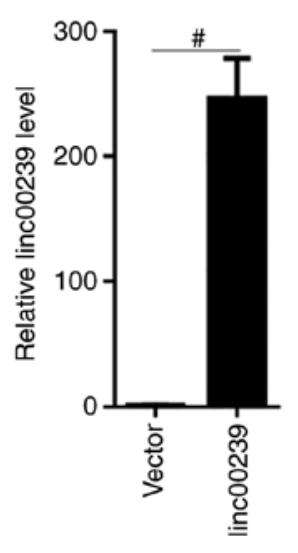

Figure 1. Detection of the expression levels of linc00239 in KG-1 and HL-60 cells (A) RT-qPCR was performed to detect the linc00239 levels in KG-1 and HL-60 cells, which were normalized to the expression levels of $\beta$-actin, GAPDH or snoU6. (B) Northern blotting was performed to detect the existence of linc00239 in KG-1 and HL-60 cells. (C) After introduction of siRNA or expression vector separately, the difference in linc00239 levels was detected by RT-qPCR. "P<0.05 vs. shScrambled-transfected KG-1 cells; ${ }^{*} \mathrm{P}<0.05$ vs. vector-transfected HL-60 cells. linc00239, long intergenic non-protein coding RNA 239.

cells, overexpression of linc00239 significantly increased the $\mathrm{IC}_{30}$ and $\mathrm{IC}_{50}$ concentrations of doxorubicin (Fig. $3 \mathrm{~A}$ and $\mathrm{B}$, lower panel). As anticipated, overexpressed linc00239 decreased the rate of cell death after $2.68 \mu \mathrm{M}$ treatment of doxorubicin (Fig. 2B).

Doxorubicin exerts an anticancer effect mainly via induction of apoptotic cell death (32). To further confirm the effects of linc00239 on doxorubicin-induced apoptotic cell death and DNA damage, apoptotic cell death and the level of phosphorylation (Ser139) and total histone $\mathrm{H} 2 \mathrm{AX}$ protein by Annexin V-FITC/PI double staining were assessed, followed by flow cytometric assay and western blotting. These data revealed that in KG-1, knockdown of linc00239 significantly increased the proportion of Annexin $\mathrm{V}^{-F_{T C C}} / \mathrm{PI}^{-}$and Annexin V-FITC ${ }^{+} \mathrm{PI}^{+}$(Fig. 4A). Since KG-1 cells presented a much higher expression level of linc00239 compared to HL-60 cells, linc00239 was knocked down in KG-1 and overexpressed linc00239 in HL-60 cells. In HL-60 cells, overexpression of linc00239 significantly decreased the proportion of Annexin V-FITC ${ }^{+} / \mathrm{PI}^{-}$. Detection of phosphorylation (Ser139) and total histone $\mathrm{H} 2 \mathrm{AX}$ protein illustrated that the overexpression of linc00239 inhibited phosphorylation (Ser139) of histone H2AX without disturbing total histone H2AX (Fig. 4B). To elucidate whether linc00239 had a protective effect against DNA damage in KG-1 and HL-60 cells, a terminal dUTP nick-end labelling (TUNEL) assay was performed. The results clearly revealed a decrease in the percentage of TUNEL-positive cells, confirming the protective effect against doxorubicin-induced DNA damage (Fig. 4C).

The presence of linc00239 is tightly associated with activation of the PI3K/Akt/mTOR pathway. To assess whether the expression of linc00239 was associated with activation of the $\mathrm{PI} 3 \mathrm{~K} / \mathrm{Akt} / \mathrm{mTOR}$ pathway, the phosphorylation of Akt and mTOR was detected after linc00239 knockdown in KG-1 cells or overexpression in HL-60 cells. Forty-eight hours after transfection, protein lysates were obtained and analysed by western blotting. The results revealed that knockdown of linc00239 reduced the phospho/total Akt ratio in KG-1 cells, and in contrast, overexpression of linc00239 significantly increased the phospho/total Akt ratio in L-60 (Fig. 5A). Moreover, the expression level of linc00239 also positively regulated the phosphorylation levels of mTOR on S2448, thus affecting mTORC1 activity, without disturbing mTOR total protein. To further confirm whether linc00239 regulated the $\mathrm{PI} 3 \mathrm{~K} / \mathrm{Akt} / \mathrm{mTOR}$ pathway by promoting phosphorylation of Akt and mTOR, the dual PI3K/Akt and mTOR inhibitor NVP-BEZ235 was employed. After pretreatment with $0.5 \mu \mathrm{M}$ NVP-BEZ235, phosphorylation of Akt and mTOR were inhibited, and the phosphorylation-promoting effect of linc00239 was abolished (Fig. 5B). 
A

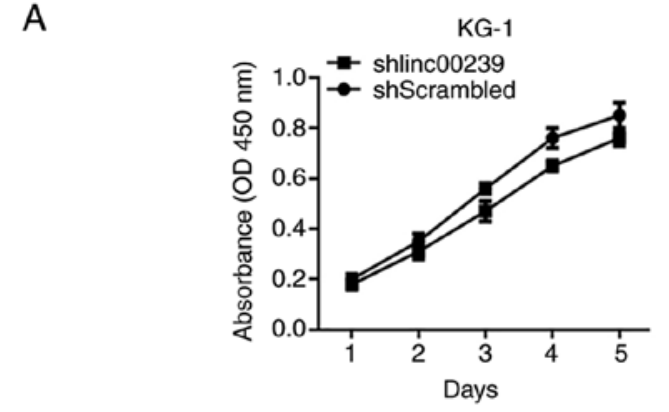

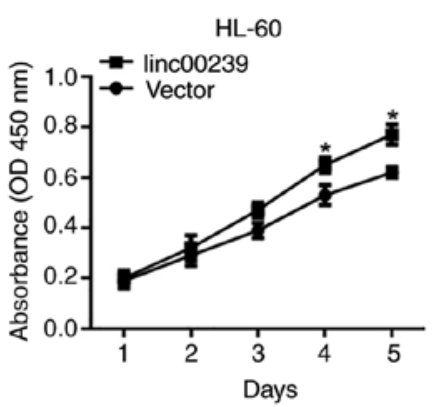

B
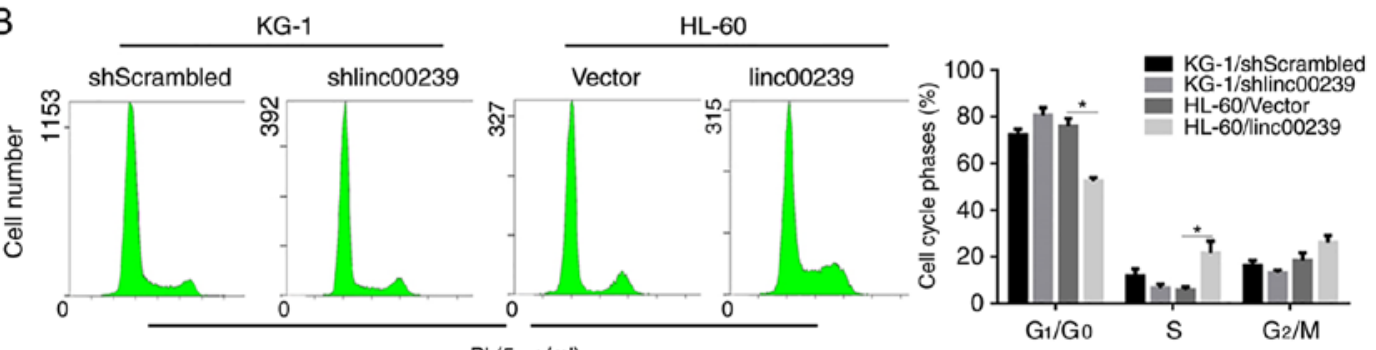

C

Pl $(5 \mu \mathrm{g} / \mathrm{ml})$
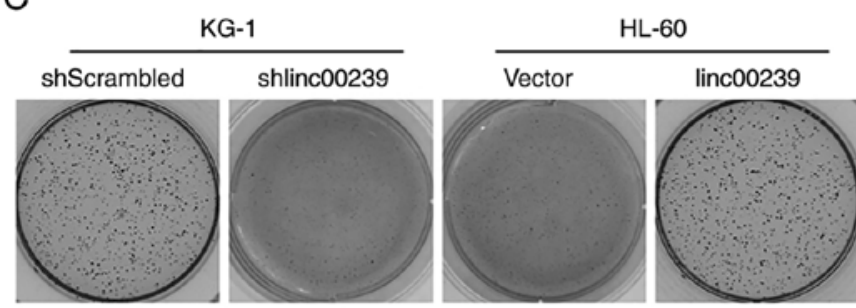

D

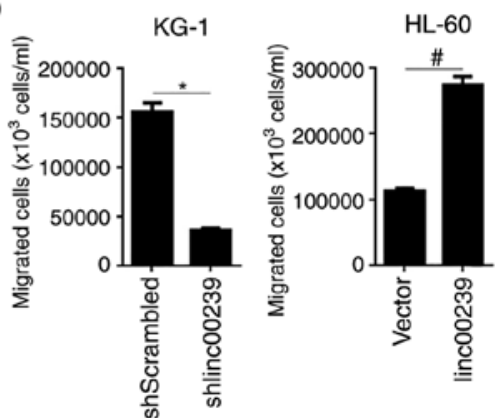

Figure 2. Effects of linc00239 on proliferation, colony formation, cell cycle distribution, migration and apoptosis. (A) A CCK-8 assay was performed to assess the effect of modified linc00239 in KG-1 or HL-60 cells, respectively. "P<0.05 vs. vector-transfected HL-60 cells. (B) The effects of knockdown or overexpression of linc 00239 on cell cycle phases. ${ }^{*} \mathrm{P}<0.05$ vs. vector-transfected HL-60 cells. (C) Colony formation in soft agar was performed after knockdown or overexpression of linc00239. (D) A migration assay was performed to detect the effects of expressed linc00239 on cell migration. "P<0.05 vs. shScrambled-transfected KG-1; ${ }^{*} \mathrm{P}<0.05$ vs. vector-transfected HL-60 cells. linc00239, long intergenic non-protein coding RNA 239; CCK-8, Cell Counting Kit-8.
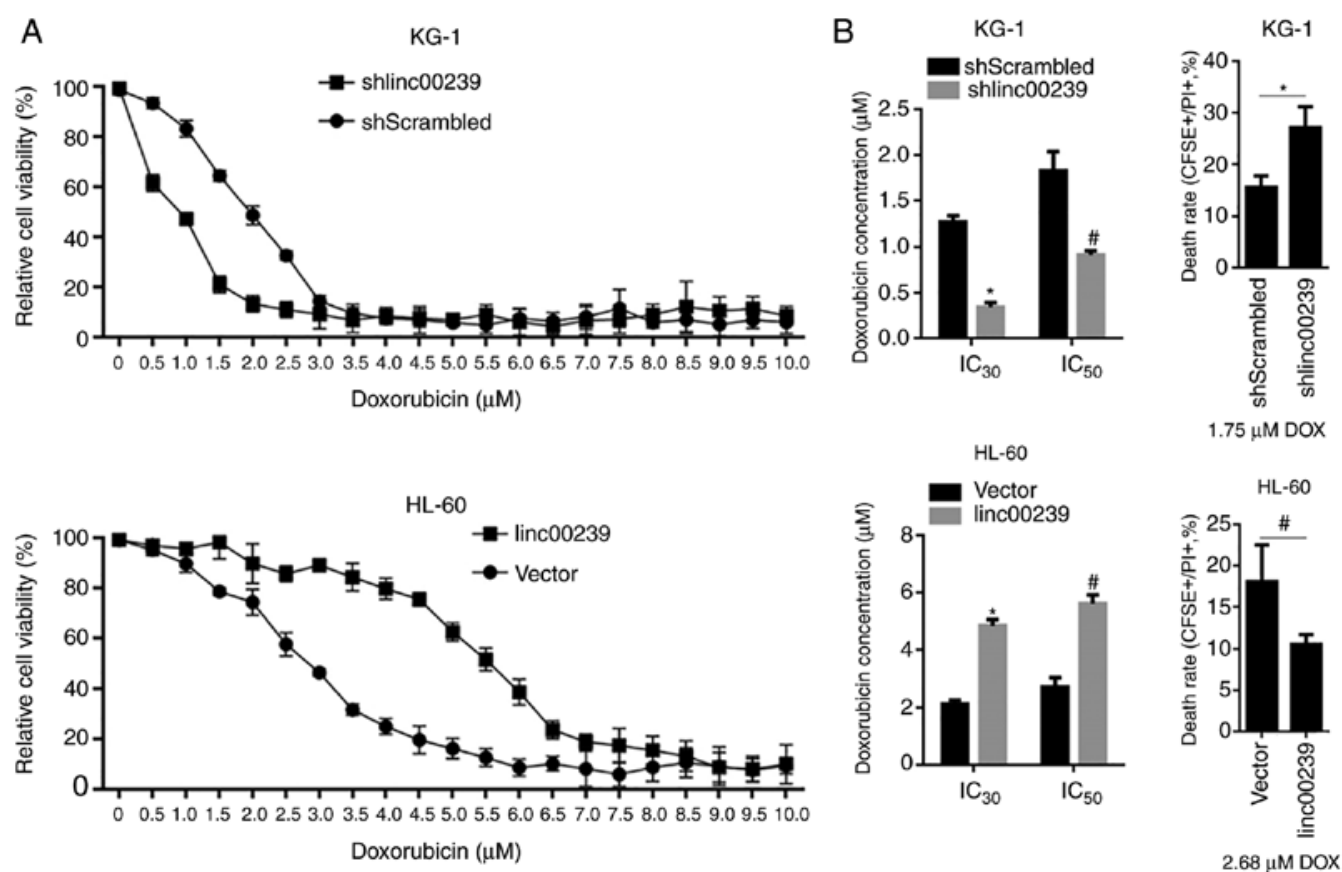

Figure 3. Effects of linc00239 on the chemosensitivity of KG-1 or HL-60 to doxorubicin. Chemosensitivity to doxorubicin was measured by the (A) cell viability assay, and $(\mathrm{B}) \mathrm{IC}_{30}$ and $\mathrm{IC}_{50}$ were assessed separately. ${ }^{*} \mathrm{P}<0.05$ vs. shScrambled-transfected KG-1 cells; ${ }^{*} \mathrm{P}<0.05$ vs. vector-transfected HL-60 cells. linc00239, long intergenic non-protein coding RNA 239. 
A
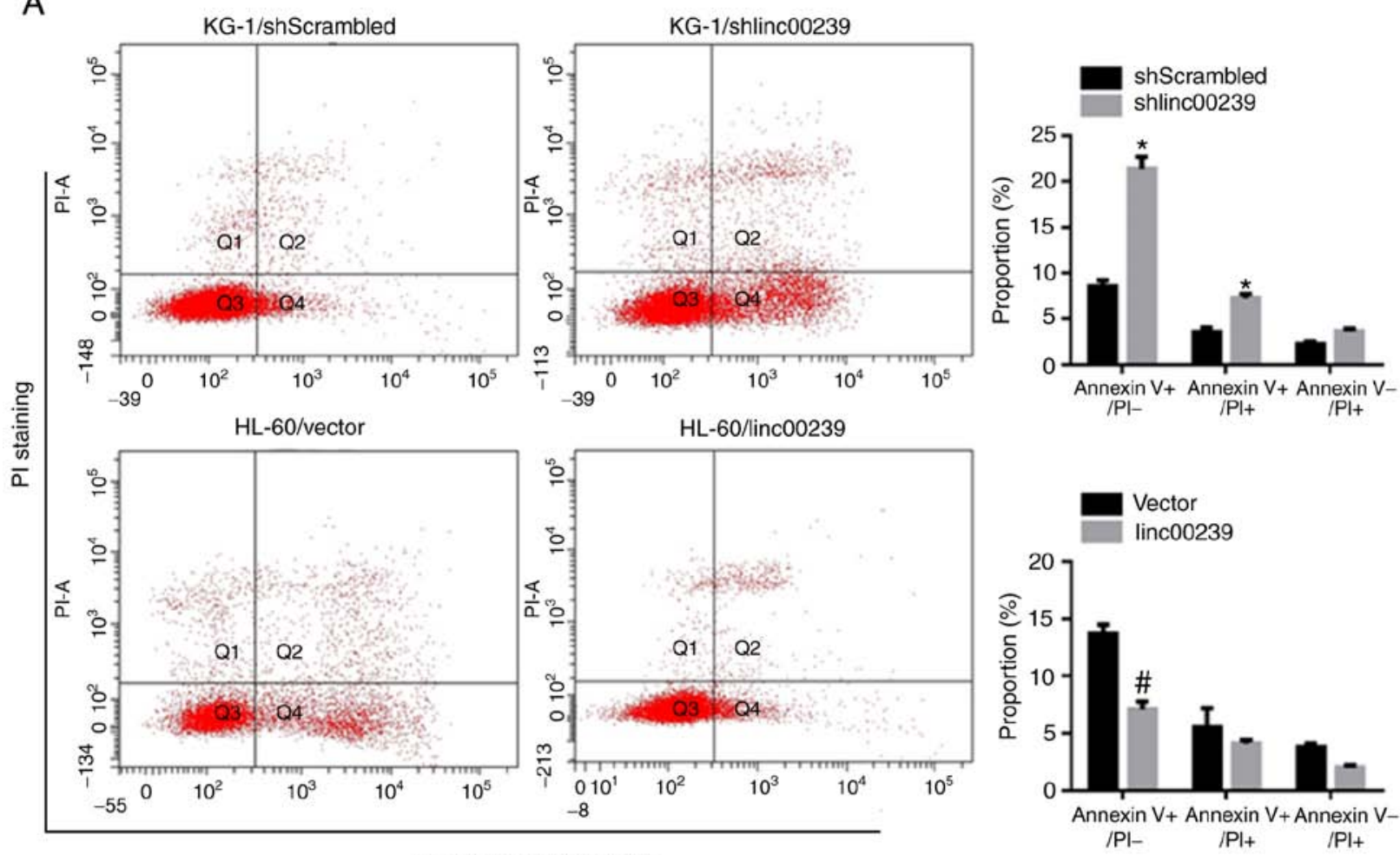

Annexin V- FITC staining

B

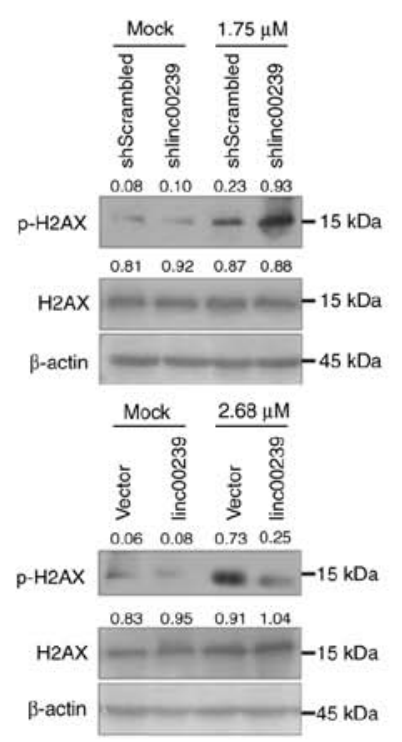

C

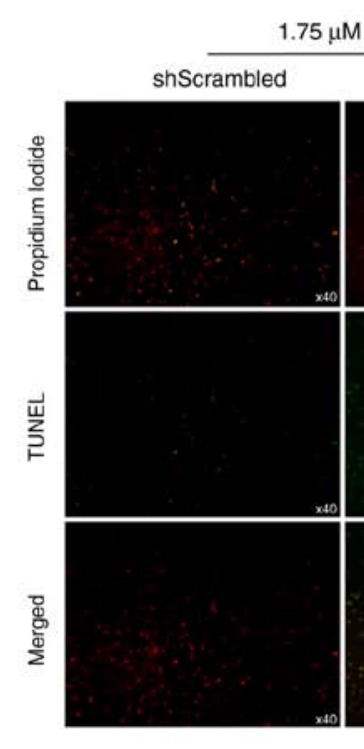

$5 \mu \mathrm{M} \mathrm{DOX}$

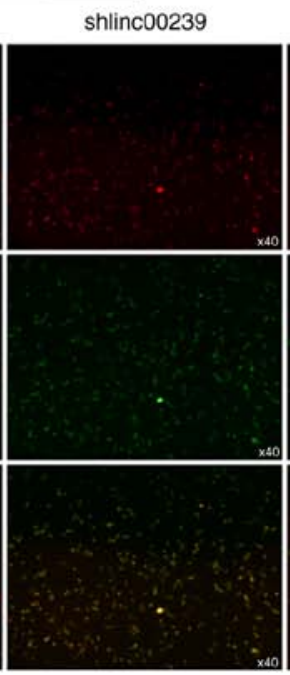

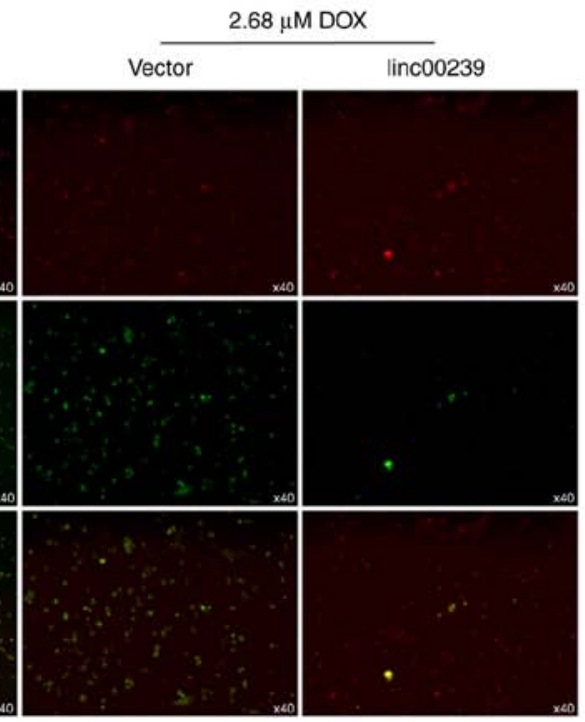

Figure 4. Downregulated or upregulated linc00239 affects doxorubicin-induced apoptotic cell death. (A) Annexin V-FITC/PI double staining was performed, followed by flow cytometric analysis. ${ }^{*} \mathrm{P}<0.05$ vs. shScrambled-transfected KG-1 cells; ${ }^{\#} \mathrm{P}<0.05$ vs. vector-transfected HL-60 cells. (B) Western blotting was performed to detect H2AX and phosphorylated H2AX after doxorubicin treatment. (C) The TUNEL assay was performed to visualize apoptotic cells after doxorubicin treatment. linc00239, long intergenic non-protein coding RNA 239; PI, propidium iodide.

Effects of linc00239 on malignant behaviours, including proliferation, colony formation, migration and chemosensitivity, are abolished by NVP-BEZ235 treatment. As aforementioned (Fig. 5), activation of the PI3K/Akt/mTOR pathway by linc00239 was eliminated by NVP-BEZ235 treatment. To demonstrate whether linc00239 functioned via regulation of the $\mathrm{PI} 3 \mathrm{~K} / \mathrm{Akt} / \mathrm{mTOR}$ pathway and thus regulated malignant behaviours in AML cells, the effects of linc00239 on proliferation, colony formation, migration, and chemosensitivity were detected after the PI3K/Akt/mTOR pathway was inhibited by NVP-BEZ235 treatment in HL-60 cells. As anticipated, after NVP-BEZ235 pretreatment, the regulatory effects of linc00239 on the proliferation, colony formation, migration and chemosensitivity to doxorubicin were eliminated (Fig. 6). All of these data indicated that linc00239 regulated malignant behaviours mainly via activation of the PI3K/Akt/mTOR pathway. 

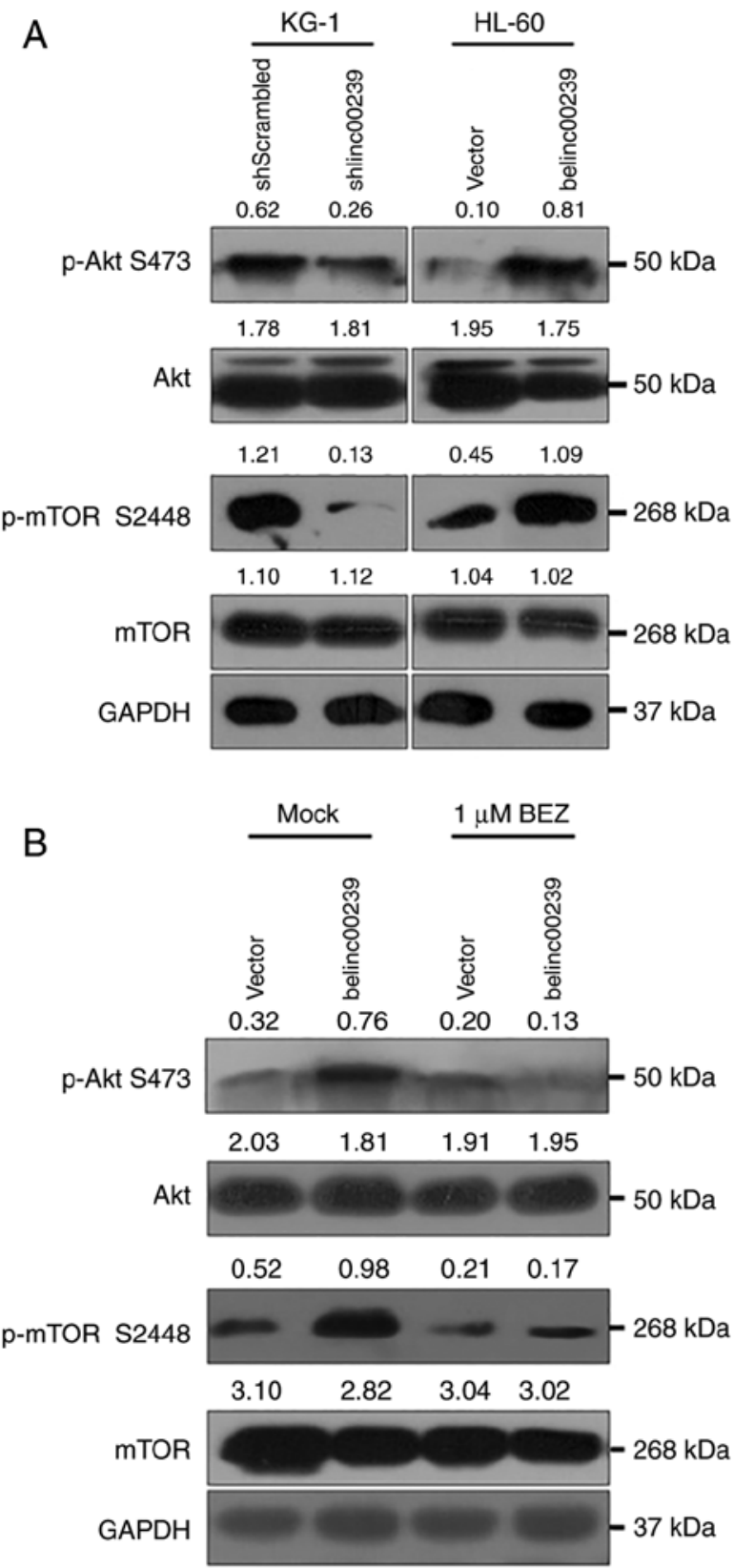
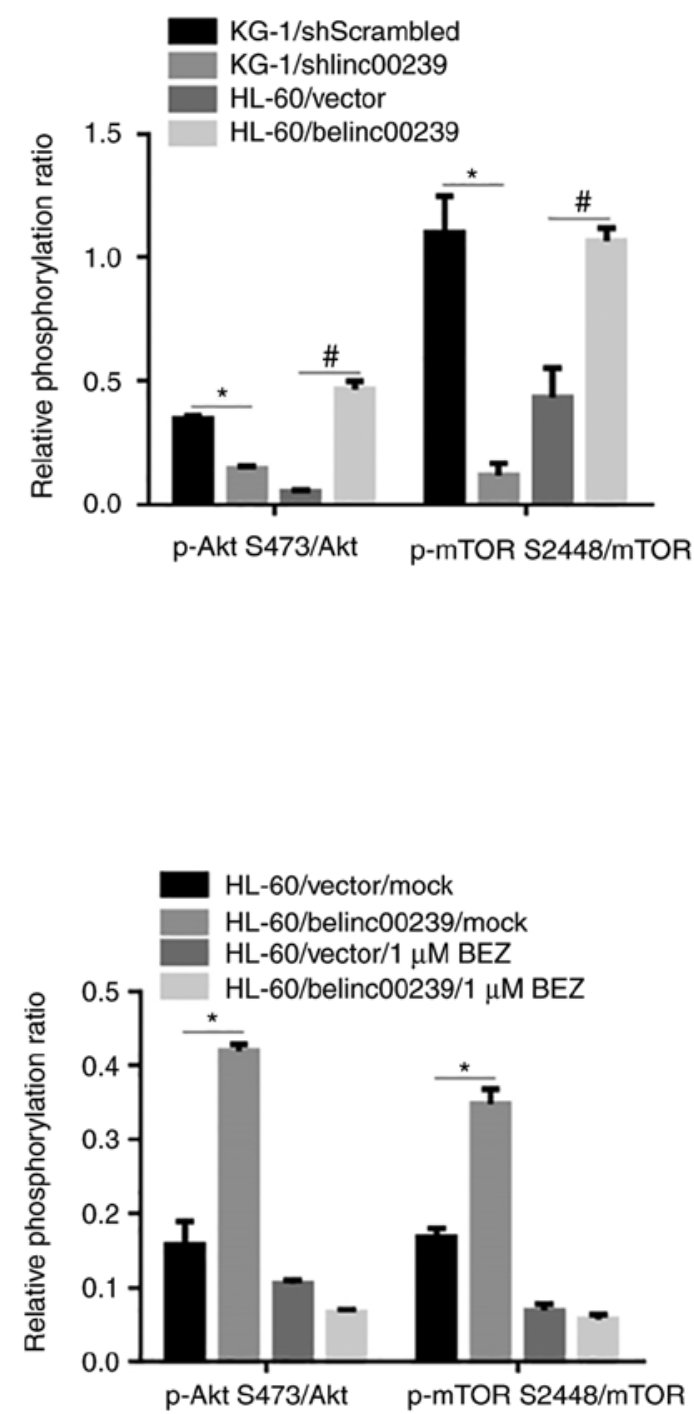

Figure 5. linc00239 contributes to PI3K/Akt/mTOR pathway activation in both KG-1 and HL-60 cells. (A) After introduction of shlinc00239 or linc00239 expression vectors, the total amount of Akt, mTOR, or phosphorylated Akt and mTOR was assessed. ${ }^{*} \mathrm{P}<0.05$ vs. shScrambled-transfected KG-1 cells; ${ }^{*} \mathrm{P}<0.05$ vs. vector-transfected HL-60 cells. (B) After $1 \mu \mathrm{M}$ BEZ treatment, the activating effects of ectopic linc00239 on the PI3K/Akt/mTOR pathway were assessed by western blotting. " $\mathrm{P}<0.05$ vs. mock treated HL-60 cells/vector. linc00239, long intergenic non-protein coding RNA 239; BEZ, NVP-BEZ235.

\section{Discussion}

In the present study, by performing RT-qPCR and northern blotting in the AML cell lines KG-1 and HL-60, we revealed the existence of linc00239 RNA in these two cell lines was revealed, which was an expected result. In order to compare the relative amount of linc00239 in these two cell lines, RT-qPCR was performed, and the data were normalized to $\beta$-actin mRNA, GAPDH mRNA, or snoU6 RNA. These results indicated that the endogenous linc00239 level in KG-1 cells was much higher than that of HL-60 cells. The expression difference between these cell lines allowed us to investigate the roles of linc00239 in the malignant behaviours of AML. To achieve this purpose, KG-1 cells were used to downregulate linc00239; HL-60 cells were used to overexpress linc00239. In this way, it was revealed that the presence of linc00239 in AML cells was positively related to malignant behaviours, including proliferation, colony formation and migration.

Doxorubicin, a cytotoxic antiproliferative and apoptosis-inducing drug, was clinically and widely used as a cytotoxic agent for chemotherapy in several types of cancers, including AML $(33,34)$. It disturbs the DNA replication processes, resulting in cell cycle arrest and cellular apoptosis (35). Thus, the effects of linc00239 on doxorubicin-induced cell cycle arrest and apoptotic cell death were investigated. Notably, without an evident effect on cell cycle arrest (data not shown), the presence of linc00239 significantly desensitized KG-1 and HL-60 cells to doxorubicin. To confirm 
A

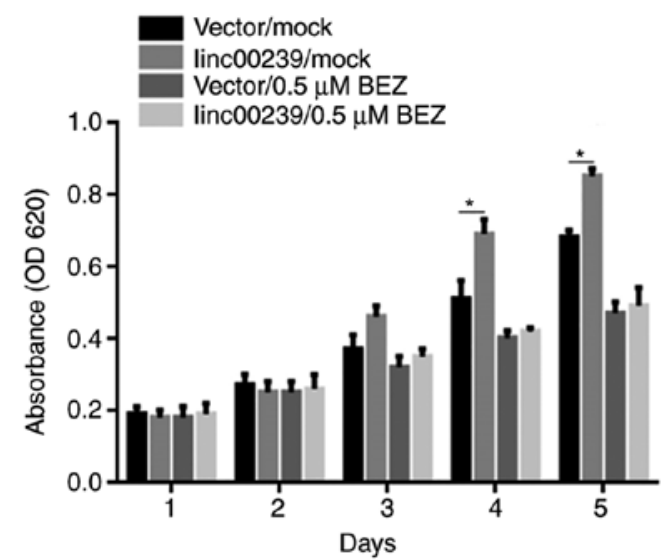

B

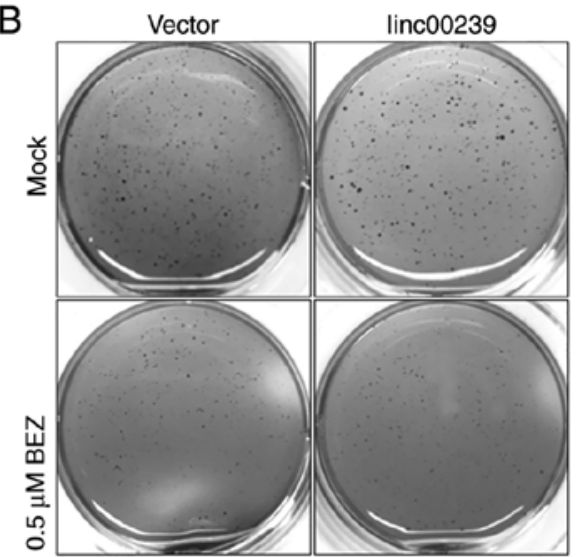

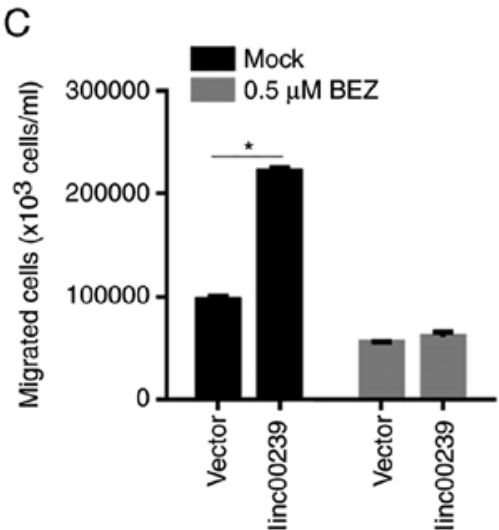

$\mathrm{E}$

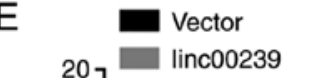

D

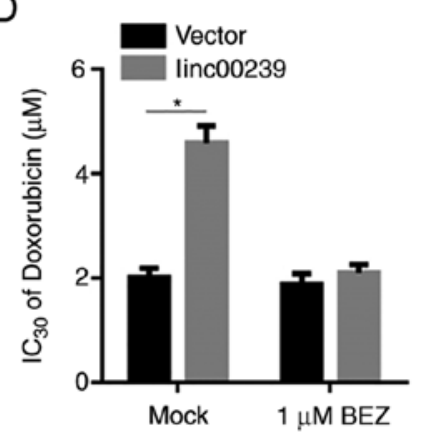

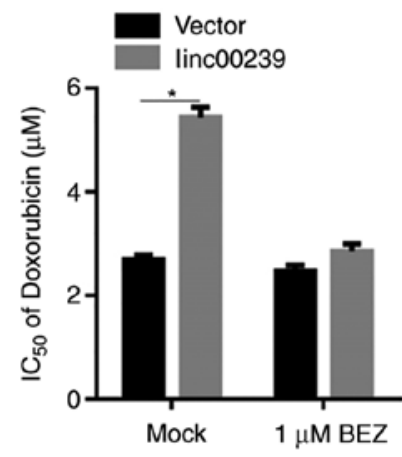

$\mathrm{F}$

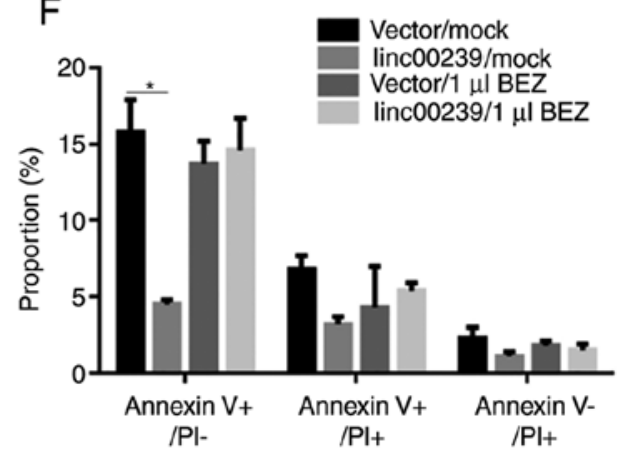

Figure 6. linc00239 promotes malignant behaviours mainly via activating the PI3K/Akt/mTOR pathway. After $0.5 \mu \mathrm{M}$ NVP-BEZ235 treatment, proliferation (A), colony formation (B), and migration (C) were detected. (D) Vector or linc00239-expressing vector-transfected HL-60 cells were pre-treated with $1 \mu \mathrm{M}$ BEZ for $24 \mathrm{~h}$ and were detected by the CCK-8 assay to measure chemosensitivity. ${ }^{*} \mathrm{P}<0.05$ vs. mock treated HL-60/vector. (E) CFSE/PI double staining was performed to detect the cell death rate after $2.68 \mu \mathrm{M}$ doxorubicin treatment. ${ }^{*} \mathrm{P}<0.05$ vs. mock treated HL-60/vector. (F) Annexin V-FITC/PI double-staining was performed to detect the apoptotic cell death rate after $2.68 \mu \mathrm{M}$ doxorubicin treatment. ${ }^{*} \mathrm{P}<0.05 \mathrm{vs}$. mock treated HL-60/vector. linc00239, long intergenic non-protein coding RNA 239; BEZ, NVP-BEZ235; CCK-8, Cell Counting Kit-8; carboxyfluorescein diacetate succinimidyl ester; PI, propidium iodide.

the effects of linc00239 on chemosensitivity to doxorubicin, the apoptotic rate and the rate of cell death were evaluated, and the expected results were obtained. No obvious effects of linc00239 on cell cycle distribution were due to the induced cell death. Moreover, the existence of linc00239 also affected doxorubicin-induced cell death in an unknown manner.

It was also revealed that the presence of linc00239 was tightly related to the phosphorylation of AKT at S473 and mTOR at S2448, without affecting total protein levels. After pretreatment with an inhibitor of the PI3K/AKT/mTOR pathway, BEZ235, the presence of linc00239 failed to affect the PI3K/AKT/mTOR pathway and to exert protective effects against doxorubicin treatment. Moreover, when pre-treated with BEZ235, the regulatory roles of linc00239 on malignant behaviours in KG-1 or HL-60 cells were eliminated. $\mathrm{PI} 3 \mathrm{~K} / \mathrm{AKT} / \mathrm{mTOR}$ is considered as the most important factor in initiation and maintenance of AML, and the abnormality of this pathway was found in most AML cases (36). Our data revealed that the presence of linc00239 tightly affected the activation of the PI3K/ATK/mTOR pathway, indicating that it is a potential therapeutic target in AML. Moreover, in a future investigation, it is worth studying the epigenetic regulating role of microRNA and long non-coding RNA on this pathway. 
In summary, it was revealed that linc00239 tightly regulated the activation of the PI3K/AKT/mTOR pathway without knowing the target in the PI3K/AKT/mTOR pathway, and by depending on this, it desensitized KG-1 or HL-60 cells to the chemoagent. The presence of linc00239 positively regulated the malignant behaviours, including proliferation, colony formation and migration in KG-1 and HL-60 cells. Collectively, linc00239 functioned as a PI3K/AKT/mTOR pathway activator and may be considered as a therapeutic target for AML treatment.

\section{Acknowledgements}

Not applicable.

\section{Funding}

The present study was supported by the Chengdu Scientific Benefiting Project (no. 2015-HM01-00461-SF).

\section{Availability of data and materials}

The datasets used during the present study are available from the corresponding author upon reasonable request.

\section{Authors' contributions}

YY performed the cellular and molecular experiments. WD contributed to the molecular experiments and was responsible for the data collection. YS performed the cellular experiments. $\mathrm{ZZ}$ performed the molecular experiments and was a major contributor in writing the manuscript. All authors read and approved the final manuscript.

\section{Ethics approval and consent to participate}

Not applicable.

\section{Patient consent for publication}

Not applicable.

\section{Competing interests}

The authors state that they have no competing interests.

\section{References}

1. Troy JD, Atallah E, Geyer JT and Saber W: Myelodysplastic syndromes in the United States: An update for clinicians. Ann Med 46: 283-289, 2014.

2. Daver N and Cortes J: Molecular targeted therapy in acute myeloid leukemia. Hematology 17 (Suppl 1): S59-S62, 2012.

3. Stone R, Sekeres M and Garcia-Manero G: Evolving strategies in the treatment of MDS and AML. Clin Adv Hematol Oncol 7: $1-14,2009$.

4. Buitenhuis $\mathrm{M}$ and Coffer PJ: The role of the PI3K-PKB signaling module in regulation of hematopoiesis. Cell Cycle 8: 560-566, 2009.

5. Fruman DA and Rommel C: PI3K and cancer: Lessons, challenges and opportunities. Nat Rev Drug Discov 13: 140-156, 2014.

6. Martelli AM, Evangelisti C, Chiarini F and McCubrey JA: The phosphatidylinositol 3-kinase/Akt/mTOR signaling network as a therapeutic target in acute myelogenous leukemia patients. Oncotarget 1: 89-103, 2010.
7. Altman JK, Sassano A and Platanias LC: Targeting mTOR for the treatment of AML. New agents and new directions. Oncotarget 2: 510-517, 2011.

8. Birkenkamp KU, Geugien M, Schepers H, Westra J,Lemmink HH and Vellenga E: Constitutive NF-kappaB DNA-binding activity in AML is frequently mediated by a Ras/PI3-K/PKB-dependent pathway. Leukemia 18: 103-112, 2004.

9. Xu Q, Simpson SE, Scialla TJ, Bagga A and Carroll M: Survival of acute myeloid leukemia cells requires PI3 kinase activation. Blood 102: 972-980, 2003.

10. Neri LM, Borgatti P, Tazzari PL, Bortul R, Cappellini A, Tabellini G, Bellacosa A, Capitani S and Martelli AM: The phosphoinositide 3-kinase/AKT1 pathway involvement in drug and all-trans-retinoic acid resistance of leukemia cells. Mol Cancer Res 1: 234-246, 2003.

11. Kentsis A, Topisirovic I, Culjkovic B, Shao L and Borden KL: Ribavirin suppresses eIF4E-mediated oncogenic transformation by physical mimicry of the 7-methyl guanosine mRNA cap. Proc Natl Acad Sci USA 101: 18105-18110, 2004.

12. Assouline S, Culjkovic B, Cocolakis E, Rousseau C, Beslu N, Amri A, Caplan S, Leber B, Roy DC, Miller WH Jr and Borden KL: Molecular targeting of the oncogene eIF4E in acute myeloid leukemia (AML): A proof-of-principle clinical trial with ribavirin. Blood 114: 257-260, 2009.

13. Chapuis N, Tamburini J, Green AS, Vignon C, Bardet V, Neyret A, Pannetier M, Willems L, Park S, Macone A, et al: Dual inhibition of PI3K and mTORC1/2 signaling by NVP-BEZ235 as a new therapeutic strategy for acute myeloid leukemia. Clin Cancer Res 16: 5424-5435, 2010.

14. Altman JK, Sassano A, Kaur S, Glaser H, Kroczynska B, Redig AJ, Russo S, Barr S and Platanias LC: Dual mTORC2/mTORC1 targeting results in potent suppressive effects on acute myeloid leukemia (AML) progenitors. Clin Cancer Res 17: 4378-4388, 2011.

15. Park S, Chapuis N, Bardet V, Tamburini J, Gallay N, Willems L, Knight ZA, Shokat KM, Azar N, Viguié F, et al: PI-103, a dual inhibitor of Class IA phosphatidylinositide 3-kinase and mTOR, has antileukemic activity in AML. Leukemia 22: 1698-1706, 2008.

16. Zeng Z, Shi YX, Tsao T, Qiu Y, Kornblau SM, Baggerly KA, Liu W, Jessen K, Liu Y, Kantarjian H, et al: Targeting of mTORC1/2 by the mTOR kinase inhibitor PP242 induces apoptosis in AML cells under conditions mimicking the bone marrow microenvironment. Blood 120: 2679-2689, 2012.

17. Papa V, Tazzari PL, Chiarini F, Cappellini A, Ricci F, Billi AM, Evangelisti C, Ottaviani E, Martinelli G, Testoni N, et al: Proapoptotic activity and chemosensitizing effect of the novel Akt inhibitor perifosine in acute myelogenous leukemia cells. Leukemia 22: 147-160, 2008.

18. Huarte M: The emerging role of lncRNAs in cancer. Nat Med 21: 1253-1261, 2015.

19. Iyer MK, Niknafs YS, Malik R, Singhal U, Sahu A, Hosono Y, Barrette TR, Prensner JR, Evans JR, Zhao S, et al: The landscape of long noncoding RNAs in the human transcriptome. Nat Genet 47: 199-208, 2015.

20. Garzon R, Volinia S, Papaioannou D, Nicolet D, Kohlschmidt J, Yan PS, Mrózek K, Bucci D, Carroll AJ, Baer MR, et al: Expression and prognostic impact of lncRNAs in acute myeloid leukemia. Proc Natl Acad Sci USA 111: 18679-18684, 2014.

21. Wu S, Zheng C, Chen S, Cai X, Shi Y, Lin B and Chen Y: Overexpression of long non-coding RNA HOTAIR predicts a poor prognosis in patients with acute myeloid leukemia. Oncol Lett 10: 2410-2414, 2015.

22. Mer AS, Lindberg J, Nilsson C, Klevebring D, Wang M, Grönberg H, Lehmann S and Rantalainen M: Expression levels of long non-coding RNAs are prognostic for AML outcome. J Hematol Oncol 11: 52, 2018.

23. Zhu Y, Zhang X, Qi L, Cai Y, Yang P, Xuan G and Jiang Y: HULC long noncoding RNA silencing suppresses angiogenesis by regulating ESM-1 via the PI3K/Akt/mTOR signaling pathway in human gliomas. Oncotarget 7: 14429-14440, 2016.

24. Yao X, Yan C, Zhang L, Li Y and Wan Q: LncRNA ENST00113 promotes proliferation, survival, and migration by activating $\mathrm{PI} 3 \mathrm{~K} / \mathrm{Akt} / \mathrm{mTOR}$ signaling pathway in atherosclerosis. Medicine 97: e0473, 2018.

25. Trimarchi T, Bilal E, Ntziachristos P, Fabbri G, Dalla-Favera R, Tsirigos A and Aifantis I: Genome-wide mapping and characterization of Notch-regulated long noncoding RNAs in acute leukemia. Cell 158: 593-606, 2014. 
26. Venter JC, Adams MD, Myers EW, Li PW, Mural RJ, Sutton GG, Smith HO, Yandell M, Evans CA, Holt RA, et al: The sequence of the human genome. Science 291: 1304-1351, 2001.

27. LivakKJ and SchmittgenTD: Analysis of relative gene expression data using real-time quantitative PCR and the $2^{-\Delta \Delta C_{\mathrm{T}}}$ method. Methods 25: 402-408, 2001.

28. Bhan A and Mandal SS: Long noncoding RNAs: Emerging stars in gene regulation, epigenetics and human disease. ChemMedChem 9: 1932-1956, 2014.

29. Wapinski $\mathrm{O}$ and Chang HY: Long noncoding RNAs and human disease. Trends Cell Biol 21: 354-361, 2011.

30. Liu Z, Sun M, Lu K, Liu J, Zhang M, Wu W, De W, Wang Z and Wang R: The long noncoding RNA HOTAIR contributes to cisplatin resistance of human lung adenocarcinoma cells via downregualtion of $\mathrm{p} 21^{\mathrm{WAF} 1 / \mathrm{CIP} 1}$ expression. PLoS One 8: e77293, 2013.

31. Hou Z, Xu C, Xie H, Xu H, Zhan P, Yu L and Fang X: Long noncoding RNAs expression patterns associated with chemo response to cisplatin based chemotherapy in lung squamous cell carcinoma patients. PLoS One 9: e108133, 2014.
32. Naci D, El Azreq MA, Chetoui N, Lauden L, Sigaux F, Charron D, Al-Daccak R and Aoudjit F: $\alpha 2 \beta 1$ integrin promotes chemoresistance against doxorubicin in cancer cells through extracellular signal-regulated kinase (ERK). J Biol Chem 287: 17065-17076, 2012.

33. Lagadinou ED, Ziros PG, Tsopra OA, Dimas K, Kokkinou D, Thanopoulou E, Karakantza M, Pantazis P, Spyridonidis A and Zoumbos NC: c-Jun N-terminal kinase activation failure is a new mechanism of anthracycline resistance in acute myeloid leukemia. Leukemia 22: 1899-1908, 2008.

34. Kweon SH, Song JH and Kim TS: Resveratrol-mediated reversal of doxorubicin resistance in acute myeloid leukemia cells via downregulation of MRP1 expression. Biochem Biophys Res Commun 395: 104-110, 2010.

35. Tao J, Lu Q, Wu D, Li P, Xu B, Qing W, Wang M, Zhang Z and Zhang W: microRNA-21 modulates cell proliferation and sensitivity to doxorubicin in bladder cancer cells. Oncol Rep 25: 1721-1729, 2011.

36. Fransecky L, Mochmann LH and Baldus CD: Outlook on $\mathrm{PI} 3 \mathrm{~K} / \mathrm{AKT} / \mathrm{mTOR}$ inhibition in acute leukemia. Mol Cell Ther 3: $2,2015$. 